\title{
Surface-enhanced Raman spectroscopy study of 4-ATP on gold nanoparticles for basal cell carcinoma fingerprinted detection
}

\author{
Luu Manh Quynh ${ }^{1}$, Nguyen Hoang Nam ${ }^{1,2, *}$ K. Kong ${ }^{3}$, Nguyen Thi Nhung${ }^{1}$, I. Notingher ${ }^{3}$, M. \\ Henini $^{3}$ and Nguyen Hoang Luong ${ }^{2}$
}

${ }^{1}$ Faculty of Physics, Hanoi University of Science, Vietnam National University, Hanoi, 334 Nguyen Trai, Hanoi, Vietnam

${ }^{2}$ Nano and Energy Center, Hanoi University of Science, Vietnam National University, Hanoi, 334

Nguyen Trai, Hanoi, Vietnam

${ }^{3}$ School of Physics and Astronomy, Nottingham University, University Park, Nottingham NG7

2RD, UK

*Corresponding author: namnh@ hus.edu.vn

\begin{abstract}
The surface-enhanced Raman (SER) signal of 4-aminothiolphenol (4-ATP) based on the surface of gold nanoparticles colloids with size distributed from 2 to $5 \mathrm{~nm}$ were used as labeling agent to detect basal cell carcinoma (BCC) of skin. The enhanced Raman band at $1075 \mathrm{~cm}^{-1}$ corresponding to C-S stretching vibration in 4-ATP was observed during attachement to the surface of gold nanoparticles. The frequency and intensity of this band did not change when the colloids were conjugated with BerEP4 antibody which specifically binds to BCC. We show the feasibility of imaging BCC by SERS scanning the $1075 \mathrm{~cm}^{-1}$ band to detect the distribution of 4-ATP-attached gold nanoparticles attached to skin tissue ex-vivo.
\end{abstract}

Keywords: Skin cancer, basal cell carcinoma, surface-enhanced Raman scattering, gold nanoparticles. 


\section{Introduction}

Skin cancer is the most common of all types of cancers in human and its incidence rate is increasing [1]. About $74 \%$ of skin cancer cases worldwide are basal cell carcinomas (BCCs) [2]. The most efficient treatment for "high-risk" BCCs (i.e. BCCs on face and neck, or recurrent BCCs) is Mohs micrographic surgery (MMS) [3]. This procedure maximizes the removal of tumor cells, while sparing as much healthy tissue as possible. Although MMS provides improved treatment outcome compared to other treatment options, the need for a pathologist or specialized surgeons to diagnose frozen-sections during surgery has limited the widespread use of MMS, leading to cases of inappropriate inferior treatment. Frozen-section histopathology also requires laborious and time-consuming procedures, increasing the costs compared to standard excision of BCC.

Raman spectroscopic imaging is a promising technique for diagnosis of skin cancers due to its high sensitivity to molecular and structural changes associated with cancer. The use of Raman spectroscopy to detect biochemical alteration in skin tissue caused by BCCs was first demonstrated by Gniadecka et al. [4]. Raster scanning Raman spectral mapping was used to image BCC in tissue samples ex-vivo MMS [5,6]. Nevertheless, raster scanning Raman mapping requires long data acquisition times, typically days for tissue specimens of $1 \mathrm{~cm}$ x $1 \mathrm{~cm}$. More recently, multimodal spectral imaging based on tissue auto-fluorescence and Raman spectroscopy was used to reduce diagnosis time for BCC to only 30-60 minutes, which becomes feasible for use during MMS $[7,8]$.

An alternative technique that could reduce data acquisition and BCC diagnosis times during MMS is surface-enhanced Raman spectroscopy (SERS). It was discovered that in the very close vicinity of metal nanostructures, strongly increased Raman scattering signals can be obtained which are mainly due to resonances between optical fields and the collective oscillations of the free electrons in a metal. Since the discovery of this surface-enhanced Raman (SER) scattering in 1974 [9], SER scattering has been recognized as a powerful technique for biomedical applications. The application of SER scattering has been studied in cancer detection [10-12], widely in molecular structure analysis [13-16]. For non-labeling agent probes, the Raman spectra were analyzed by measuring the intrinsic signals to distinguish between healthy and diseased regions [10,11]. In these studies, SER signals of cancer-specified bio-molecules were reported as indicators that could give information about the presence of cancer gene [10] and cancer expression [11]. However, the signals were still broadened and still showed disadvantage on distinguishing the cancer and non-cancer areas. It was noticed that the SER peaks of some linkages that were close to the metal surface were strong, individually sharp and did not change, 
while the metal-organic complex being attached to other organisms or molecules. In this study, we investigated SER signal of 4-aminothiophenol (4-ATP, sometimes called p-aminothiophenol - PATP) linked to surface of gold nanoparticles while being conjugated with the skin carcinomas cell antibody BerEP4. With the conjugation with this BCC-specified antibody, SER signals of some linkages from the 4-ATP organic molecules were noticed to be stable and potentially allow detection of the tumor regions. Further, the usefulness of these SERS probes for detection of BCC in ex-vivo specimens is investigated.

\section{Experiments and methods}

\subsection{Synthesizing and coating gold nanoparticles with 4-ATP (Au-4ATP)}

Gold nanoparticles with sizes from 2 to $5 \mathrm{~nm}$ were prepared by wet chemical method using cetyltrimethylamonium bromide (CTAB - MERCK 99\%). In particularly, ion $\mathrm{Au}^{3+}$ from chloroauric acid $\left(\mathrm{HAuCl}_{4}-\mathrm{MERCK} 99 \%\right)$ was prepared in 2-times distilled water. We prepared $75 \mathrm{ml}$ of CTAB $0.2 \mathrm{M}$ and 0.2 $\mathrm{ml}$ of $\mathrm{HAuCl}_{4} 0.5 \mathrm{M}$ in a $200 \mathrm{ml}$ flask, then 2-times distilled water was filled to have $100 \mathrm{ml}$ of $1 \mathrm{mM} \mathrm{HAuCl}_{4}$ in 0.15M CTAB. We used sodium borohydride $\left(\mathrm{NaBH}_{4}-\mathrm{MERCK} 99 \%\right) 0.1 \mathrm{M}$ to reduce the dark yellow $\mathrm{Au}^{3+}$ ion containing solution to dark brown solution. After $12 \mathrm{~h}$, the color of the solution changed to dark red. 4-ATP $10^{-3} \mathrm{M}$ (MERCK 99\%) was injected to the solution with 1:40 volume rate. After $12 \mathrm{~h}$, the solution was washed several times by centrifugation. The result solution is later called Au-4ATP solution.

The structural and morphological properties of the Au-4ATP sample were investigated by X-ray diffractometer (XRD) Brucker D5005 and transmission electron microscope (TEM) JEOL JEM1010.

\subsection{Conjugation of Au-4ATP with BerEP4 (Au-4ATP-antibody)}

An amount of $3 \mathrm{mg}$ EDC was mixed with $1 \mathrm{mg}$ BerEP4 and then was added into the Au-4ATP solution. The mixture was incubated at least 20 min until the Au-4ATP totally reacted with BerEP4 molecules.

\subsection{Skin tissue samples}

Skin tissue sections were obtained from the Nottingham University Hospitals National Health Service Trust. Tissue sections were cut from blocks removed during operation and sliced into one third thickness. Two of three tissue slides were investigated by optical microscope by bright field imaging with and without conventional hematoxylin and eosin $(\mathrm{H} \& \mathrm{E})$ staining. The third slide was treated with Au-4ATP-antibody complex before being investigated by Raman microspectroscopy.

\subsection{Raman spectroscopic measurements}


Raman spectroscopic measurements were carried out by using a home-made Raman microspectrometer built by Notingher's group [6]. The laser power was set to $20 \mathrm{~mW}$ to avoid sample damage, scanning interval was set from $600 \mathrm{~cm}^{-1}$ to $1700 \mathrm{~cm}^{-1}$ and the integration time was set to $0.1 \mathrm{~s}$.

\subsection{SER spectra of $A u-4 A T P$ and $A u-4 A T P-a n t i b o d y$}

Two drops of Au-4ATP and Au-4ATP-antibody colloids containing solutions were deposited on the sample-holder surface. The spectra of the samples were observed respectively and were drawn in one image to see the differences.

\subsection{Scanning measurement of Au-4ATP-antibody treated tissue}

We dropped $1 \mu \mathrm{L}$ of Au-4ATP-antibody containing solution onto the surface of the third tissue sample. After waiting $5 \mathrm{~min}$ for the antibody conjugation with the cells the scanning measurement was set up. SER scattering signal of every single $1 \mu \mathrm{m} \times 1 \mu \mathrm{m}$ on $40 \mu \mathrm{m} \times 40 \mu \mathrm{m}$ region of the tissue sample was observed.

There were 1600 spots. All 1600 spectra from 1600 spots were collected and were analyzed by two methods. First method was principle component analysis [17]. In second method, we consider the peak at 1075 $\mathrm{cm}^{-1}$, which corresponds to stretching band of C-S linkage from 4-ATP molecules. Peak heights at $1075 \mathrm{~cm}^{-1}$ were mapped depending on the position of single spots. This landscape image then was discussed as fingerprinted image in comparison with normal bright field images of two first samples and with the image prepared by principle component analysis method.

\section{Results and discussion}

\subsection{Structure and morphology of gold nanoparticles}

Figure 1 illustrates the XRD pattern and TEM image of as-prepared gold nanoparticles. The XRD peaks at 38.2, 44.4 and 64.7 degree indicate the (111), (200) and (220) reflecting phases of fcc structure,

respectively. The calculated lattice parameter was $4.08 \pm 0.05 \AA$, which agreed with early works [18,19]. Using Debye-Scherrer formula the particle size of $4.2 \mathrm{~nm}$ was determined, in good agreement with the results observed in TEM image, where most of the particles distributed at $4 \mathrm{~nm}$.

\subsection{Gold nanoparticles surface modification}

Figure 2 shows the schematic reaction between the Au-4ATP and the carboxyl group $(-\mathrm{COOH})$ from the antibody BerEP4. Under catalytic effect of EDC the free amino group $\left(-\mathrm{NH}_{2}\right)$ of the Au-4ATP colloid reacted with the carboxyl group and a peptide group (-NH-CO) was created. 
This reaction made a stable covalent linkage that binds the gold nanoparticles with the antibody to form Au4ATP-antibody.

The Raman spectra of raw 4-ATP $10^{-3} \mathrm{M}$ and of Au-4ATP are observed (data not shown). A little shifts of the peaks are experienced as the 4-ATP molecules being deposited on Au nanoparticles' surface, which corresponds to the association of the molecules with the metal particles via Au-S bond. Besides, there is importance magnification of Raman intensity is detected in the spectrum of Au-4ATP in comparison with the spectrum of raw 4-ATP. Due to our result, the major intensity enhancement is approximately $10^{5}$-times. Due to the reaction shown in Fig. 2, some vibration modes corresponding to the $-\mathrm{NH}_{2}$ disappear and could be replaced by the vibrations of the peptide linkage, which is majority occur in BerEP4 molecules. The electromagnetic field surrounding the metal nanoparticles was enhanced from the Surface Plasmon Resonance (SPR) effect, which increased the Raman signal of the vibrations near the particles' surface [20,21]. The significant increase of the Raman signal in the SER scattering brings the idea to use it to detect the surface change of each colloids solution after linking the antibody with the gold nanoparticles. When one $-\mathrm{NH}$ linkage from the free $\mathrm{NH}_{2}$ was exchanged and then large BerEP4 molecules touched onto surface of the gold nanoparticles, some SER peaks that contain $-\mathrm{NH}$ vibrations disappeared and some peaks that characterize the peptide link appear as shown in Fig. 3.

The SER spectra of Au-4ATP and Au-4ATP-antibody containing samples are shown in Figure 3. Obviously, the measured Raman peaks at $1495 \mathrm{~cm}^{-1}, 1432 \mathrm{~cm}^{-1}$ and $1134 \mathrm{~cm}^{-1}$ of Au-4ATP disappeared after antibody was conjugated. It was discussed that these peaks are assigned to $v \mathrm{CC}+\delta \mathrm{CH}, v \mathrm{CC}+\delta \mathrm{CH}$ and $\delta \mathrm{CH}$ vibrations, respectively, where $v$ denotes stretching movement and $\delta$ bending movement $[22,23]$. We suggest that the wagging vibration $\mathrm{NH}_{2}$ linkage $\left(\pi \mathrm{NH}_{2}\right)$ might occur together with these vibrations. The disappearance of $\pi \mathrm{NH}_{2}$ vibration due to the reaction described in Fig. 2 might be responsible for the disappearance of peaks at $1495 \mathrm{~cm}^{-1}, 1432 \mathrm{~cm}^{-1}$ and $1134 \mathrm{~cm}^{-1}$. We also observed that a peak at $1382 \mathrm{~cm}^{-1}$ disappeared after the antibody conjugation. This peak was assigned to $\delta \mathrm{CH}+v \mathrm{CC}$ vibration modes $[22,23]$. The change of the $-\mathrm{CN}$ - linkage to peptide linkage (-NH-CO-) might be responsible for the disappearance of this peak. Besides, new peaks arise at $1449 \mathrm{~cm}^{-1}$ and $1297 \mathrm{~cm}^{-1}$. The peak at $1449 \mathrm{~cm}^{-1}$ is assigned to $\mathrm{CH}_{2}, \mathrm{CH}_{3}$ deformation, and the peak at 1297 $\mathrm{cm}^{-1}$ is assigned to the vibration of helix structure of amide III linkage [24]. It should be noted that Raman peaks below $1005 \mathrm{~cm}^{-1}$ were not considered because in this region, the peaks might also correspond to the phonon vibrations of the metal material. 
Enhanced Raman spectra of 4-ATP on Au-substrate was also investigated by Peter Owens' groups when they were conjugated with anti-p53 protein [27]. The characteristic peak of C-S linkage close to $1080 \mathrm{~cm}^{-1}$ was also employed as detecting signal. When the 4-ATP-modified Au surface was covalently connected with anti-p53 molecules, the peak position corresponding to the C-S vibration observed at $1080 \mathrm{~cm}^{-1}$ shifted to higher wave number value within $1 \mathrm{~cm}^{-1}$. After protein-antibody interaction, the peak position shifted about $1 \mathrm{~cm}^{-1}$ depending on the added protein concentration. The same observation is experienced in our Raman investigation.As revealed from Fig. 3, a strongest peak at $1075 \mathrm{~cm}^{-1}$ is observed, which is assigned to $v C S$ vibration, while a strong peak at $1614 \mathrm{~cm}^{-1}$ is assigned to $v C C$ vibration [22,23]. While considering $1614 \mathrm{~cm}^{-1}$ peak, people may be easily confused by other organic molecules because $v \mathrm{CC}$ vibrations are quite common for organic systems $[6,24]$. The sharp individual peak at around $1075 \mathrm{~cm}^{-1}$ was detected by Zheng et al. on SERS spectrum of 4-ATP absorbed on a silver surface [15], by Osawa et al. on SERS spectrum of 4-ATP absorbed on a silver film [22], by Jiao et al. on SERS spectrum of 4-ATP on Au surface [23,25]. Furthermore, the peak at $1075 \mathrm{~cm}^{-1}$ was not detected on the SERS spectra of antibody and/or polypeptide on metal substrates [24,26]. We propose the enhanced Raman peak at $1075 \mathrm{~cm}^{-1}$ as a strong typical signal to detect positions and concentration of Au-4ATP nanoparticles, and hence, of antibody molecules.3.3. Fingerprinted landscape of BCC tissue

As mentioned in Section 2, skin tissue sections obtained from the Nottingham University Hospitals National Health Service Trust were cut from blocks removed during operation and sliced into one third thickness. Two of three tissue slides were investigated by optical microscope by bright field imaging with and without conventional hematoxylin and eosin (H\&E) staining. The third slide was treated with Au-4ATPantibody complex as described in Section 2 before being investigated by Raman microspectroscopy. SER scattering signal of every single $1 \mu \mathrm{m} \times 1 \mu \mathrm{m}$ on $40 \mu \mathrm{m} \times 40 \mu \mathrm{m}$ region of the tissue sample was observed and analyzed by two methods. The first one was principle component analysis [17], in which the SER spectra were compared to the averaged spectrum then the difference was shown in the landscape. In second method, the peak at $1075 \mathrm{~cm}^{-1}$ corresponds to stretching band of C-S linkage from 4-ATP molecules was considered. Peak heights at $1075 \mathrm{~cm}^{-1}$ were mapped depending on the position of single spots. The fingerprinted landscape of SER signals of Au-4ATP-antibody on a BCC tissue was shown in Fig. 4.

In this work, simple H\&E staining was used as control diagnosis and Fig. 4A shows the colored image of the tissue. In this non-specific method which is being employed in Nottingham University Hospitals National Health Service Trust, immunofluorescence labeling process have not been used and only the 
condensate regions on the tissue sample were considered, where the cancer cell area may be the dark colored regions, for example, region marked by red circle A1, A2. However, the diagnostic result really depends on the subjective decision of the pathologists because this method may lead to misinterpretation of distinguishing the cancer regions and non-cancer regions. As can be seen in Fig. 4B which shown the bright-field microscopic result - with this selected sample - it is easy to confirm that the B1 region corresponds to a hair-hole position, and $\mathrm{B} 2$ is not, while $\mathrm{B} 1$ and $\mathrm{B} 2$ have the same position on the tissue with the regions marked by A1 and A2 in Fig. 4A. It is shown that the dark colored A1 regions can be misinterpretative. The result of SER signal analyzed using Principle component analysis, where the SER signal were compared with each other then the difference of the SER spectra from the average spectrum, is shown in Fig. 4C. In this lanscape, the yellow to the red colored areas such as $\mathrm{C} 1$ and $\mathrm{C} 2$ areas can be considered as cancer regions. The regions $\mathrm{C} 1$ and $\mathrm{C} 2$ have the same position with regions A1 and A2, as well as B1 and B2, respectively. Figure 4D shows the result of the SER signal analyzed using only the intensity of SER peaks at $1075 \mathrm{~cm}^{-1}$ and the regions D1 and D2 also have the same position with regions $\mathrm{C} 1$ and $\mathrm{C} 2$. In this method, the antigen-antibody coupling oriented the $\mathrm{Au}-$ 4ATP-antibody colloids close to the BCC surface and the carcinomas sections should considered as a dock where distributed high concentration of Au-4ATP-antibody particles, then the SER peak intensity at $1075 \mathrm{~cm}^{-1}$ will higher in these areas. In Fig. 4D, one can see the results of using the peak height at $1075 \mathrm{~cm}^{-1}$ to mapping the Au-4ATP-antibody appeared areas in $40 \mu \mathrm{m} \times 40 \mu \mathrm{m}$ region. However, the area D1 in figure 4D do not show the high intensity of the peak at $1075 \mathrm{~cm}^{-1}$ while the others such as D2 area indicate very high intensity of the peak at $1075 \mathrm{~cm}^{-1}$.

Anike Nijisen and co-workers used Raman spectra for BCC discrimination [28] and so did the group of Ioan Notingher [7]. In the articles, the authors reported the differences in Raman spectra of the infected and healthy regions. The total intensity of the Raman spectrum of BCC infected region was higher than that of the healthy region, which was discussed to correspond to the higher accumulation of lipids and nucleic acids within the cancer cells. From the calculation of total intensity, a scanning images of the skin tissues were made, which were employed to distinguish the infected parts to healthy parts. Without selective detector, the Raman spectra only selected the lipid- and nucleotide-condensed regions from the others, which might lead to the misinterpretation if the healthy cells also have condensed organic organism; such as in the skin hole-close region shown in our experiment. Besides, no specific peaks would be applicable for a selective discrimination of BCC tissue from healthy tissue, which lead to long acquisition time for intraoperative diagnostic (5-20 $\left.\mathrm{h} / \mathrm{mm}^{2}\right)[7]$. 
From all the results described above, only regions marked by A2, B2, C2 and D2 can be surely considered as the cancer areas, while A1, B1, C1 and D1 regions may assigned as the position of a hair-hole where the cell concentration also higher than others parts. By principle component analysis, only those regions were highlighted which differ from other regions and the non-carcinomas can also be observed, which may lead to the misinterpretation. Furthermore, the whole SER map collecting time should be longer than $2 \mathrm{~h}$ according to the collecting time of each spectrum was nearly $5 \mathrm{~s}$ while the fingerprinted image using peak height at $1075 \mathrm{~cm}^{-1}$ can be observed in around $5 \mathrm{~min}$ because the acquisition could be focused only on the narrow band around the $1075 \mathrm{~cm}^{-1}$ peak (e.g. narrow filter) rather than collect the entire spectrum, the integration time for each pixel may decrease to $0.1-0.2 \mathrm{~s}$. Hence, this method can be the solution for quick diagnostic during operation.

\section{Conclusion}

In conclusion, we were succeeded using SER signal of C-S link vibration at $1075 \mathrm{~cm}^{-1}$ on gold nanoparticles to detect BCC contaminated parts of skin tissue samples. The gold nanoparticles after being coated with 4-ATP were conjugated with BerEP4 antibody, which specifically recognize BCC. By fingerprint method using the SER peak at $1075 \mathrm{~cm}^{-1}$, the image of $40 \mu \mathrm{m} \times 40 \mu \mathrm{m}$ skin sample was obtained, and showed the position of the tumors. These SERS probes show promise for fast and selective diagnosis of BCC by collecting the fingerprinted spectral image of skin resections. Furthermore, all the results can be observed and analyzed automatically without requiring subjective interpretation by pathologists.

\section{References}

1. J. M. Baxter, A. N. Patel, and S. Varma, BMJ 345, e5342. (2012)

2. National Cancer Intelligence Network (NCIN). Non-melanoma skin cancer in England, Scotland, Northern Ireland, and Ireland. London, NCIN, 2013.

3. S. V. Mohan and A. L. S. Chang, Curr. Dermatol. Rep. 3, 40. (2014)

4. M. Gniadecka, H.C. Wulf, O.F. Nielsen, D.H. Christensen, and J. Hercogova, Photochem. Photobiol. 66, 418. (1997)

5. A. Nijssen, T. C. Bakker Schut, F. Heule, P. J. Caspers, D. P. Hayes, M. H. A. Neumann, and G. J. Puppels, J. Invest. Dermatol. 119, 64. (2002) 
6. M. Larraona-Puy, A. Ghita, A. Zoladek, W. Perkins, S. Varma, I.H. Leach, A.A. Koloydenko, H. Williams, and I. Notingher, J. Biomed. Opt. 14, 054031. (2009)

7. K. Kong, C. J. Rowlands, S. Varma, W. Perkins, I. H. Leach, A. A. Koloydenko, H. C. Williams, and I. Notingher, Proc. Natl. Acad. Sci. U.S.A. 110, 15189. (2013)

8. S. Takamori, K. Kong, S. Varma, I. Leach, H.C. Williams, and I. Notingher, Biomedical Optics Express 6, 98. (2015)

9. M. Fleischmann, P.J. Hendra, and A.J. McQuillan, Chem. Phys. Lett. 26, 163. (1974)

10. T. Vo-Dinh, L.R. Allain, and D.L. Stokes, J. Raman Spectrosc. 33, 511. (2002)

11. P.M. Kasili, M.B. Wabuyele, and T. Vo-Dinh, NanoBiotechnology 2, 29. (2006)

12. L.R. Allain and T. Vo-Dinh, Analyt. Chim. Acta 469, 149. (2002)

13. N.J. Kim, J. Phys. Chem. C 114, 13979. (2010)

14. S. Ye, L. Fang, and Y. Lu, J. Raman Spectrosc. 41, 1119. (2010)

15. J. Zheng, Y. Zhou, X. Li, Y. Ji, T. Lu, and R. Gu, Langmuir 19, 632. (2003)

16. Y.C. Liu, Langmuir 18, 174. (2002)

17. C.M. Stellman, K.S. Booksh, A.R. Muroski, M.P. Nelson, and M.L. Myrick, Sci. Eng. Comp. Mater. 7 , 51. (1998)

18. N.N. Long, L.V. Vu, C.D. Kiem, S.C. Doanh, C.T. Nguyet, P. T. Hang, N.D. Thien, and L.M. Quynh, J. Phys.: Conf. Ser. 187, 012026. (2009)

19. X. Huang, I.H. El-Sayed, and M.A. El-Sayed, J. Am. Chem. Soc. 128, 2115. (2006)

20. H.Y.Jung, Y.K. Park, S. Park, and S.K. Kim, Anal. Chim. Acta 602, 236. (2007)

21. E. C. Le Ru, E. Blackie, M. Meyer, and P. G. Etchegoin, J. Phys. Chem. C 111, 13794. (2007)

22. M. Osawa, N. Matsuda, K. Yoshii, and I. Uchida, J. Phys. Chem. 98, 12702. (1994)

23. L.S. Jiao, L. Niu, J. Shen, T. You, S. Dong, and A. Ivaska, Electrochem. Commun. 7, 219 (2005).

24. N.C. Maiti, M.M. Apetri, M.G. Zagorski, P.R. Carey, and V.E. Anderson. J. Am. Chem. Soc. 126, 2399. (2004)

25. L.S. Jiao, Z. Wang, L. Niu, J. Shen, T. You, S. Dong, and A. Ivaska, J. Solid State Electrochem. $10,886$. (2006)

26. T. M. Herne, A. M. Ahern, and R.L. Garrell, Anal. Chim. Acta 246, 75. (1991)

27. Peter Owens, Nigel Phillipson, Jayakumar Perumal, Gerard M. O’Connor and Malini Olivo. Biosensors. 5, 664-677. (2015). 
28. Annieke Nijssen, Tom C. Bakker Schut, Freerk Heule, Peter J. Caspers, Donal P. Hayes,Martino H. A. Neumann, and Gerwin J. Puppels. The journal of investigative dermatology. 119(1), 64-69, (2002). 

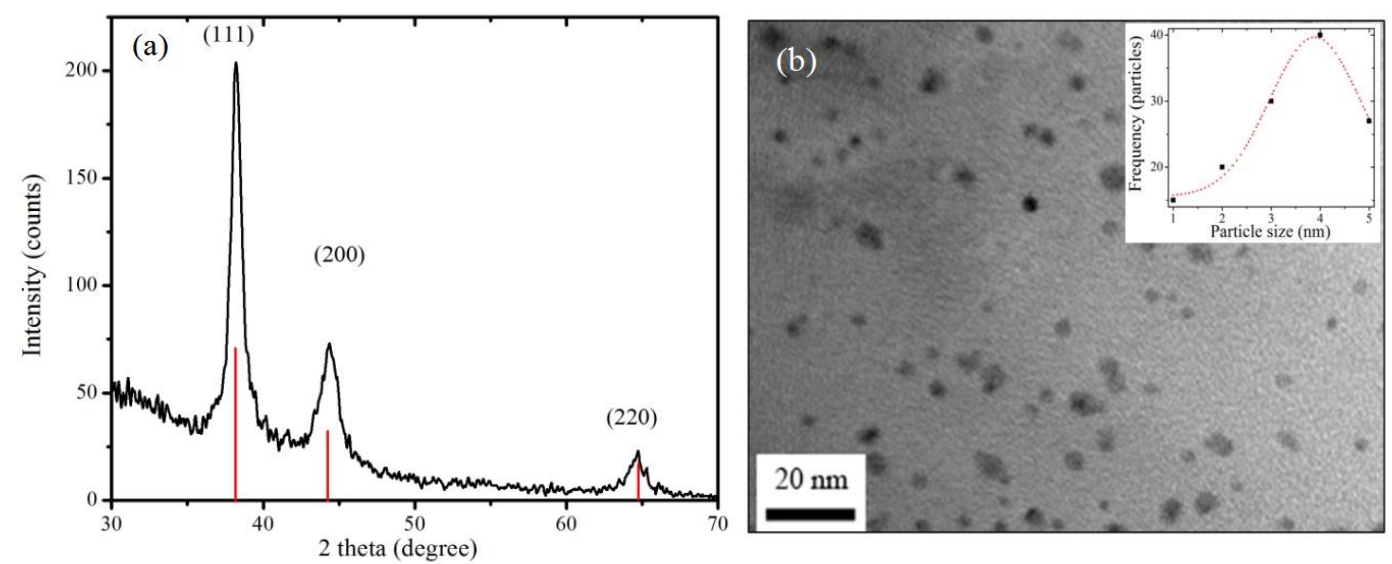

Fig. 1. (a) X-ray diffraction pattern of as-prepared gold nanoparticles. Black pattern shows the measurement data and the red vertical lines show the standard diffraction positions of (111), (200) and (220) planes of Au bulk material (pattern 4-784). (b) TEM image of as-prepared gold nanoparticles. Dark grey and black dots show the presence of the nanoparticles in the sample. Insert: size distribution of the nanoparticles calculated from the TEM image. 


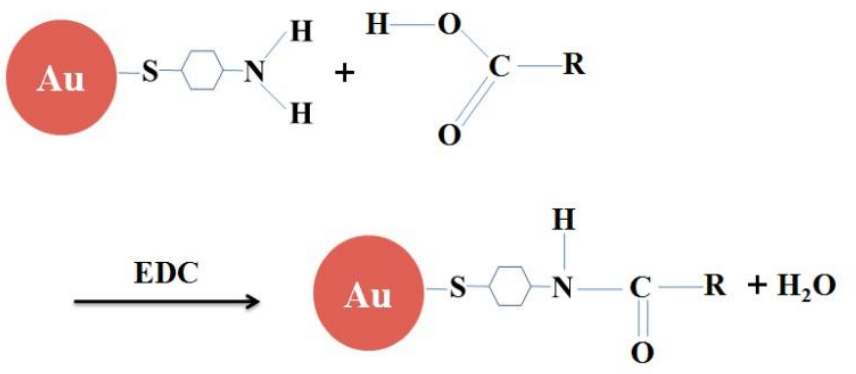

Fig. 2. Schematic graph of peptide link creating reaction. Au-S covalent link is well-known that links the 4-ATP molecules to the gold nanoparticles' surfaces and let the amino group $\left(-\mathrm{NH}_{2}\right)$ freely dissolved in solution. After reacting with the carboxyl groups $(-\mathrm{COOH})$ from the antibody BerEP4 molecules with the present catalyst EDC, peptide (-NH-CO-) binding formulates. Here the R-COOH denotes the whole antibody, of which we consider only the reaction of one carboxyl group and $\mathrm{R}$ - is the remained part. 


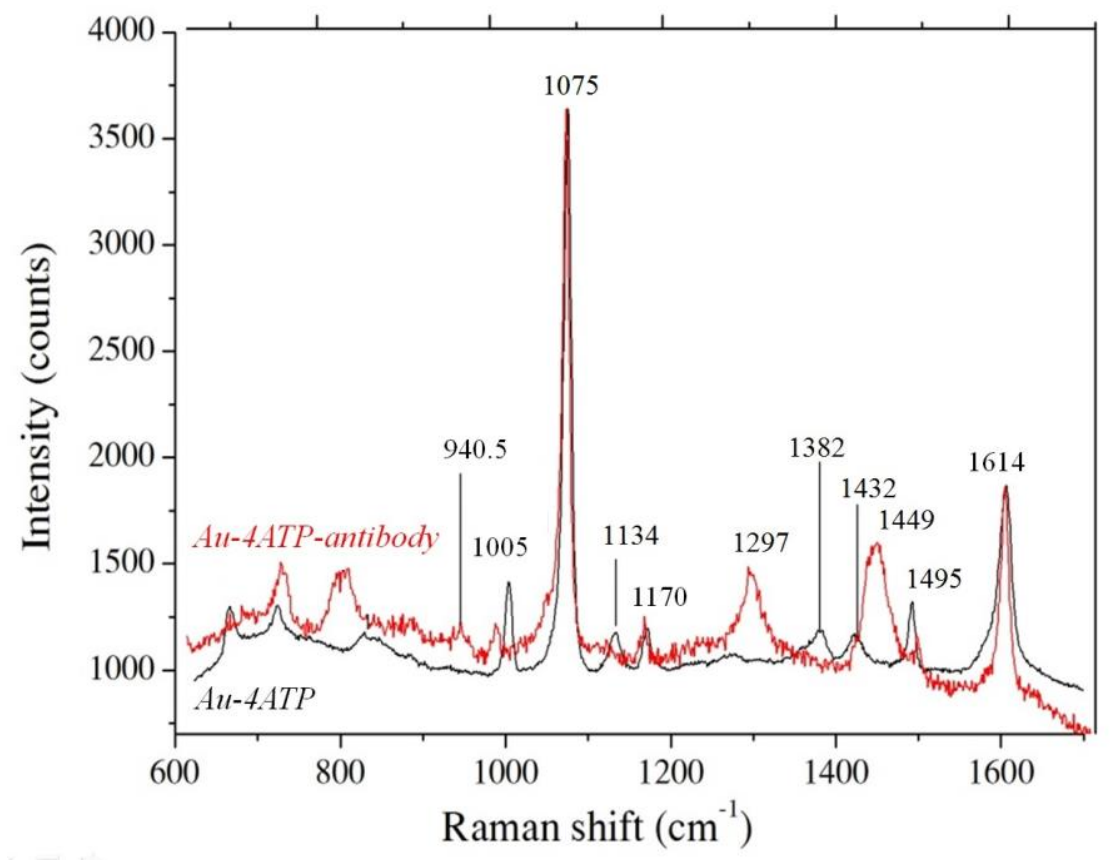

Fig. 3. SER spectra of Au-4ATP and Au-4ATP-antibody containing samples. 

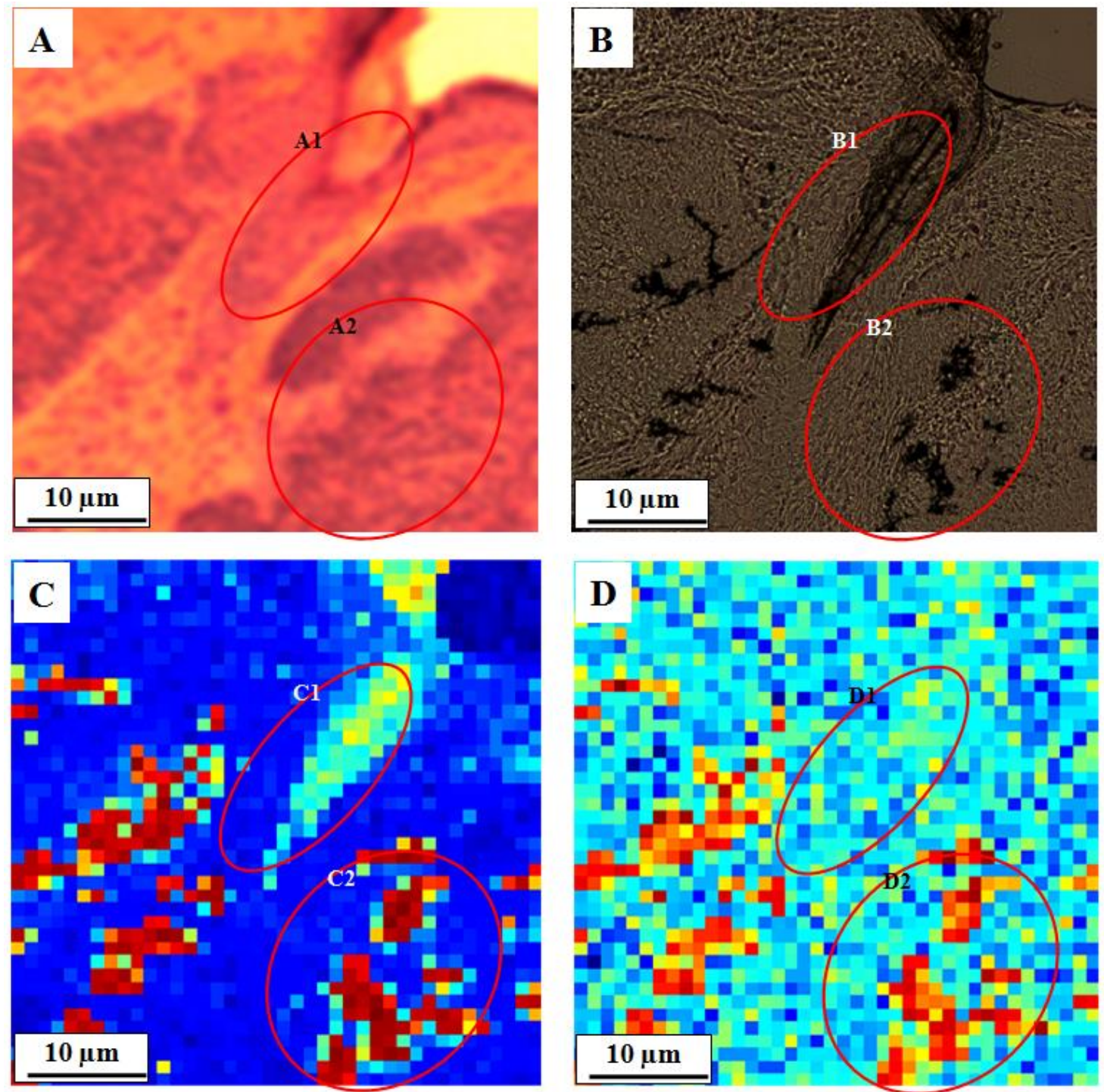

Fig. 4. Fingerprinted landscape of SER signals of Au-4ATP-antibody on BCC tissue. (A) Gram staining

picture of a BCC tissue where region marked as A1 and A2 are the suspected area; (B) Bright field Microscope picture of BCC tissue where regions B1 and B2 are the same position on the tissue with the regions A1 and A2, respectively; (C) SER signal landscape analyzed by Principle component method, where regions $\mathrm{C} 1$ and $\mathrm{C} 2$ are the same position on the tissue with regions A1 and A2, respectively; (D) The fingerprinted landscape of intensity of SER peaks at $1075 \mathrm{~cm}^{-1}$, where regions D1 and D2 are the same position on the tissue with regions A1 and A2, respectively. The difference of D1 and D2 show that only red colored D2 and the similar color area are the infected area while D1 is not. These results show that this method can be the better solution for diagnostic during operation. 